\title{
Using spectral flux ratios to standardize SN la luminosities ${ }^{\star}$
}

\author{
The Nearby Supernova Factory: \\ S. Bailey ${ }^{1}$, G. Aldering ${ }^{2}$, P. Antilogus ${ }^{1}$, C. Aragon ${ }^{2}$, C. Baltay ${ }^{3}$, S. Bongard ${ }^{1}$, C. Buton ${ }^{4}$, M. Childress ${ }^{2,5}$, N. Chotard ${ }^{4}$, \\ Y. Copin ${ }^{4}$, E. Gangler ${ }^{4}$, S. Loken ${ }^{2}$, P. Nugent ${ }^{2}$, R. Pain ${ }^{1}$, E. Pecontal ${ }^{6}$, R. Pereira ${ }^{4}$, S. Perlmutter $^{2,5}$, D. Rabinowitz ${ }^{3}$, \\ G. Rigaudier ${ }^{6}$, K. Runge ${ }^{2}$, R. Scalzo ${ }^{3}$, G. Smadja ${ }^{4}$, H. Swift ${ }^{2,5}$, C. Tao ${ }^{7}$, R. C. Thomas ${ }^{2, \star \star}$, and C. Wu ${ }^{1}$ \\ ${ }^{1}$ Laboratoire de Physique Nucléaire et des Hautes Énergies, CNRS/IN2P3; Universités Paris VI et Paris VII, 4 place Jussieu, Tour 43 , \\ 75252 Paris Cedex 05, France \\ e-mail: stephen.bailey@in2p3.fr \\ 2 Physics Division, Lawrence Berkeley National Laboratory, 1 Cyclotron Road, Berkeley, CA 94720, USA \\ 3 Department of Physics, Yale University, New Haven, CT 06250-8121, USA \\ 4 Université de Lyon, 69622, France; Université de Lyon 1, France; CNRS/IN2P3, Institut de Physique Nucléaire de Lyon, France \\ 5 Department of Physics, University of California Berkeley, Berkeley, CA 94720, USA \\ ${ }^{6}$ Université de Lyon, 69622, France; Université de Lyon 1, France; CRAL, Observatoire de Lyon, 69230 Saint Genis Laval, France; \\ CNRS, UMR 5574; ENS de Lyon, France \\ 7 CPPM, 163 Av. Luminy, 13288 Marseille Cedex 09, France
}

Received 2 March 2009 / Accepted 4 Mai 2009

\section{ABSTRACT}

\begin{abstract}
We present a new method to standardize type Ia supernova (SN Ia) luminosities to $\lesssim 0.13$ mag using flux ratios from a single fluxcalibrated spectrum per SN. Using Nearby Supernova Factory spectrophotomery of $58 \mathrm{SNe}$ Ia, we performed an unbiased search for flux ratios that correlate with SN Ia luminosity. After developing the method and selecting the best ratios from a training sample, we verified the results on a separate validation sample and with data from the literature. We identified multiple flux ratios whose correlations with luminosity are stronger than those of light curve shape and color, previously identified spectral feature ratios, or equivalent width measurements. In particular, the flux ratio $\mathcal{R}_{642 / 443}=F(642 \mathrm{~nm}) / F(443 \mathrm{~nm})$ has a correlation of 0.95 with SN Ia absolute magnitudes. Using this single ratio as a correction factor produces a Hubble diagram with a residual scatter standard deviation of $0.125 \pm 0.011 \mathrm{mag}$, compared with $0.161 \pm 0.015 \mathrm{mag}$ when fit with the SALT2 light curve shape and color parameters $x_{1}$ and $c$. The ratio $\mathcal{R}_{642 / 443}$ is an effective correction factor for both extrinsic dust reddening and instrinsic variations such as those of SN 1991T-like and SN 1999aa-like SNe. When combined with broad-band color measurements, spectral flux ratios can standardize SN Ia magnitudes to $\sim 0.12$ mag. These are the first spectral metrics that give robust improvements over the standard normalization methods based upon light curve shape and color, and they provide among the lowest scatter Hubble diagrams ever published.
\end{abstract}

Key words. stars: supernovae: general - cosmology: observations

\section{Introduction}

The ability to standardize the absolute luminosity of type Ia supernovae (SNe Ia) makes them powerful cosmological probes through their use as luminosity distance indicators. Uncorrected observations of SN Ia absolute magnitudes have an rms scatter of $\sim 40 \%$; corrections for light curve shape and color normalize them to $15 \%$ to $20 \%$, i.e., luminosity distances of SNe Ia can be measured to 7-10\% accuracy using current standardization techniques (Phillips 1993; Prieto et al. 2006; Guy et al. 2007; Jha et al. 2007). Improved standardization would reduce both statistical and systematic errors, since the remaining dispersion represents an upper limit to possible bias from uncorrected physical effects that could arise from variations in host galaxy dust properties, progenitor properties such as mass, metallicity, and age, and explosion physics such as geometry.

Spectral indicators such as feature ratios (e.g., Nugent et al. 1995; Bongard et al. 2006; Foley et al. 2008) and pseudoequivalent widths (e.g., Hachinger et al. 2006; Bronder et al. 2008) have been proposed to enhance peak SN Ia normalization

* Table 2 is only available in electronic form at http: //www . aanda.org

$\star \star$ Luis W. Alvarez Fellow. from light curve shape and color, or even as alternative approaches altogether which use a single night's observations to correct observed magnitudes (e.g., Riess et al. 1998). While these methods showed promise for what could be acheived with spectra, none of them is robustly competitive with light curve shape and color corrections alone.

In this work, we take a new unbiased approach to the problem of finding optimal spectral flux ratios for standardizing SN Ia peak brightness. Flux ratios best correlated with absolute magnitudes are detected in a training set and cross-validated on a separate subsample. Hubble diagram fits are compared using standard lightcurve color and shape corrections, using only flux ratios as standardization parameters, or using flux ratios combined with light curve color measurements. We find several new robust spectral indicators that outperform light curve shape and color corrections.

\section{Data and analysis}

This analysis uses spectrophotometry of 58 of the supernovae obtained by the Nearby Supernova Factory (SNfactory) collaboration using its SuperNova Integral Field Spectrograph (SNIFS, Aldering et al. 2002) on the University of Hawaii 2.2-m 
telescope on Mauna Kea. This subset consists of SNfactory SNe Ia for which final host-galaxy follow-up has been completed and which pass minimal cuts on the quality of the light curve fit. Targets were additionally required to have a spectrum within \pm 2.5 restframe days of $B$-band maximum light; if multiple spectra were available, the one nearest maximum light was used. Hubble residuals, colors, and light curve shape parameters were not a factor in selecting this subsample. These $\mathrm{SNe}$ are evenly distributed in the redshift range $0.02<z<0.09$, which allows relative distance measurements with minimal uncertainties from peculiar velocities. The methods were developed on a training subset of $28 \mathrm{SNe}$ before cross checking the results on a separate validation sample of $30 \mathrm{SNe}$ to ensure that the final results are not simply overfitting statistical fluctuations of the dataset.

The subsets have consistent distributions of light curve width and color, redshifts, and Hubble residuals; these are consistent with other published SNe Ia used for cosmology measurements and training lightcurve fitters. The host galaxies include spirals, ellipticals, and irregulars, and the host properties span an order of magnitude in progenitor age, metallicity, and inferred amount of dust extinction. This is comparable to the estimated evolution of the universal mean of these quantities to $z \sim 1$ (e.g., Riess \& Livio 2006; Kowalski et al. 2008) thus the results from this dataset are also likely to work well for high- $z$ SNe Ia.

The spectra were dereddened to correct for Milky Way dust (Cardelli et al. 1989; Schlegel et al. 1998), deredshifted, and rebinned in $c \Delta \lambda / \lambda \sim 2000 \mathrm{~km} \mathrm{~s}^{-1}$ bins from 350 to $850 \mathrm{~nm} \mathrm{(23} \mathrm{to}$ $56 \AA$ bins) to equally sample spectral features in the physically relevant velocity space while still over-sampling SN Ia spectral features. The exact bin sizes used were not rigorously optimized for this analysis, though cross checks were performed to ensure that the results are not sensitive to the exact binning chosen.

Photometry was synthesized from the multi-epoch fluxcalibrated spectra in box filters corresponding to approximately $B, V$, and $R$. Light curves were fit using SALT2 (Guy et al. 2007) to estimate their restframe $B$-band peak magnitudes $m_{B}$ and calculate their uncorrected $B$-band Hubble diagram residuals (or equivalently, their uncorrected absolute magnitudes). Alternatively, $m_{B}$ was synthesized directly from the spectra, independent of an SN light curve model.

The correlation of absolute magnitudes with each possible binned flux ratio was calculated for the training sample as shown in Fig. 1. The upper-left triangle of the color density plot shows the absolute Pearson correlation $\rho$ of the flux ratios $\mathcal{R}_{y / x}=F_{y} / F_{x}$ with the SN Ia absolute magnitudes; the bottom-right triangle shows the color corrected correlations as described below. Using $\log (\mathcal{R})$ produced similar though slightly worse results.

Spearman rank order correlations were also calculated in order to be less sensitive to outliers. Although the Spearman correlation is a more robust statistic, the Pearson correlation is a better predictor of the effects on a Hubble diagram $\chi^{2}$ fit since outliers affect the Pearson correlation and $\chi^{2}$ fits in similar ways. We selected the best ratios using the simple average of the Pearson and Spearman correlations; by considering both types of correlations, we select relatively strong correlations while avoiding those which are primarily due to outliers. We also checked the robustness of selected peaks by comparing the results from other near-max spectra in the training sample which were not the spectra closest to maximum light.

The statistical significance of prospective correlations in the training set was assessed using Monte Carlo permutation testing before the validation set was unblinded. For each random trial, Hubble diagram residuals were randomized and the ratio with

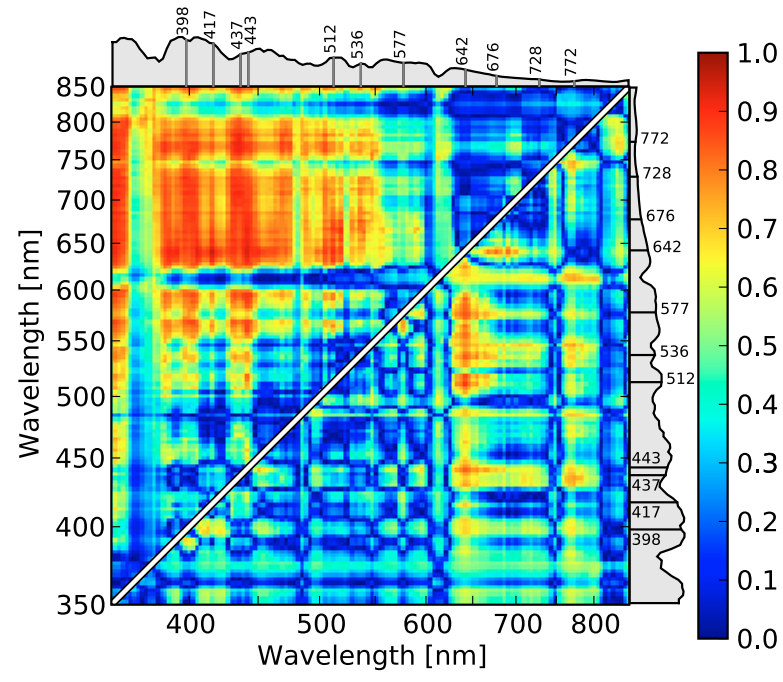

Fig. 1. Absolute Pearson correlations of flux ratios $\mathcal{R}_{y / x}=F_{y} / F_{x}$ with SN Ia absolute magnitudes. The upper-left triangle shows the correlations with uncorrected absolute magnitudes. For illustration, the lowerright triangle shows the correlations of color-corrected ratios $\mathcal{R}^{c}$ with color-corrected magnitudes. A median SN Ia spectrum is included on each axis for reference along with the wavelengths used in the flux ratios of Table 1.

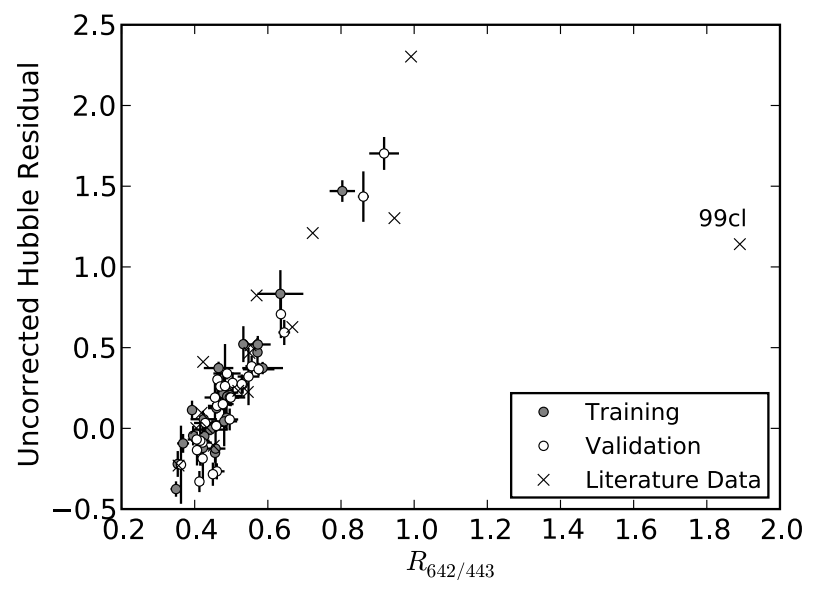

Fig. 2. Hubble diagram residuals vs. $\mathcal{R}_{642 / 443}$ for the training set (filled circles), validation set (open circles), and literature data ( $\times$ symbols).

the highest correlation was retained. We find that $\mathcal{P}(|\rho|>0.9)<$ $10^{-5}$, compared with multiple peaks above 0.9 in Fig. 1.

After selecting the five best flux ratios from the training sample, we unblinded the results for those ratios for the validation sample. Figure 2 shows the results for the highest ranked ratio, $\mathcal{R}_{642 / 443}$. It plots the uncorrected Hubble diagram residuals $\Delta \mu_{B} v s . \mathcal{R}_{642 / 443}$ for the training set of supernovae (filled circles) and validation set (open circles). The correlation is very strong (0.95), consistent between the two samples, and is not dominated by just a few supernovae. For comparison, the correlation of Hubble residuals with the combination of the SALT2 light curve shape and color parameters $x_{1}$ and $c$ is 0.92 .

We fit a Hubble diagram of the $58 \mathrm{SNe}$ using flux ratios $\mathcal{R}$ with a distance modulus of $\mu_{B}=\left(m_{B}-M^{\prime}\right)+\gamma \mathcal{R}$, where $m_{B}$ is the restframe $B$-band magnitude from either SALT2 (without any color or $x_{1}$ corrections) or directly synthesized from the spectrophotometry, $M^{\prime}$ absorbs offsets from the $\mathcal{R}$ intercept and the mean SN Ia absolute magnitude, and $\gamma$ is solved in the fit along with $M^{\prime}$ to minimize the $\chi^{2}$ of the residuals. The 
Table 1. Correlations of flux ratios $\mathcal{R}$ with SN Ia absolute magnitudes and standard deviations of Hubble diagram fits.

\begin{tabular}{ccccccccc}
\hline \hline Correction & \multicolumn{3}{c}{ Correlation with absolute magnitude } & \multicolumn{5}{c}{ Hubble diagram residual scatter } \\
parameter(s) & Training & Validation & Combined & $\gamma$ & Training & Validation & Combined & $\sigma_{\text {core }}$ \\
\hline $\mathcal{R}_{642 / 443}$ & 0.94 & 0.96 & 0.95 & $3.5 \pm 0.2$ & $0.130 \pm 0.018$ & $0.134 \pm 0.018$ & $0.128 \pm 0.012$ & 0.108 \\
$\mathcal{R}_{642 / 417}$ & 0.95 & 0.91 & 0.91 & $4.9 \pm 0.2$ & $0.114 \pm 0.016$ & $0.185 \pm 0.025$ & $0.166 \pm 0.016$ & 0.162 \\
$\mathcal{R}_{772 / 437}$ & 0.92 & 0.94 & 0.93 & $7.3 \pm 0.3$ & $0.142 \pm 0.020$ & $0.160 \pm 0.021$ & $0.152 \pm 0.014$ & 0.125 \\
$\mathcal{R}_{642 / 512}$ & 0.90 & 0.95 & 0.93 & $4.7 \pm 0.3$ & $0.162 \pm 0.022$ & $0.146 \pm 0.020$ & $0.154 \pm 0.015$ & 0.152 \\
$\mathcal{R}_{728 / 398}$ & 0.90 & 0.93 & 0.91 & $7.9 \pm 0.3$ & $0.162 \pm 0.022$ & $0.168 \pm 0.022$ & $0.172 \pm 0.016$ & 0.138 \\
\hline$c, \mathcal{R}_{642 / 519}^{c}$ & 0.96 & 0.96 & 0.96 & $3.5 \pm 0.3$ & $0.106 \pm 0.015$ & $0.129 \pm 0.018$ & $0.119 \pm 0.011$ & 0.128 \\
$c, \mathcal{R}_{577 / 642}^{c}$ & 0.95 & 0.95 & 0.95 & $-1.4 \pm 0.1$ & $0.115 \pm 0.016$ & $0.150 \pm 0.020$ & $0.135 \pm 0.013$ & 0.126 \\
$c, \mathcal{R}_{642 / 536}^{c}$ & 0.95 & 0.96 & 0.95 & $2.3 \pm 0.2$ & $0.116 \pm 0.016$ & $0.134 \pm 0.018$ & $0.125 \pm 0.012$ & 0.126 \\
$c, \mathcal{R}_{676 / 642}^{c}$ & 0.94 & 0.93 & 0.93 & $-4.2 \pm 0.5$ & $0.131 \pm 0.019$ & $0.178 \pm 0.024$ & $0.157 \pm 0.015$ & 0.163 \\
$c, \mathcal{R}_{642 / 443}^{c}$ & 0.95 & 0.96 & 0.96 & $3.2 \pm 0.3$ & $0.121 \pm 0.017$ & $0.125 \pm 0.017$ & $0.119 \pm 0.011$ & 0.104 \\
\hline$c, x_{1}$ & 0.91 & 0.93 & 0.92 & $\ldots$ & $0.154 \pm 0.022$ & $0.171 \pm 0.023$ & $0.161 \pm 0.015$ & 0.156 \\
\hline
\end{tabular}

Notes: $\gamma$ is a fit parameter in the distance modulus $\mu_{B}=\left(m_{B}-M^{\prime}\right)+\gamma \mathcal{R}$, and $\sigma_{\text {core }}=1.4826 \times \operatorname{median}\left(\left|\Delta \mu_{B}-\operatorname{median}\left(\Delta \mu_{B}\right)\right|\right)$.

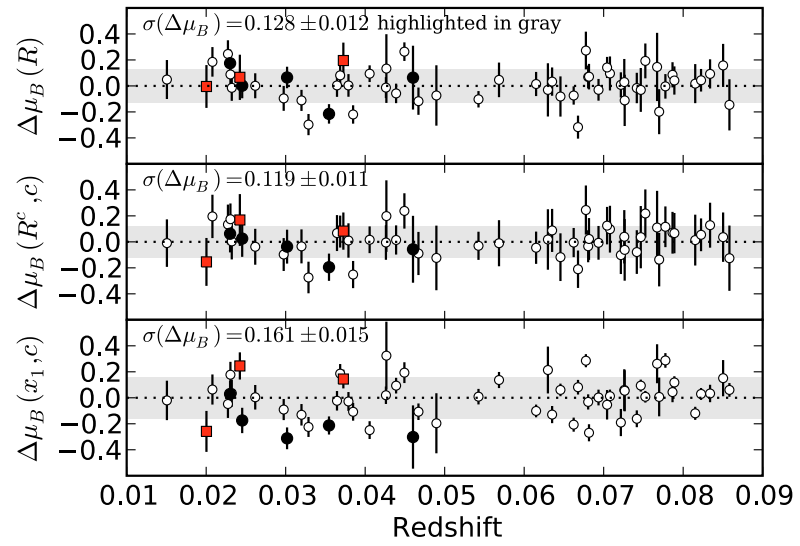

Fig. 3. Hubble diagram residuals when fit with the single parameter $\mathcal{R}_{642 / 443}$ (top), with color and dereddened ratio $\mathcal{R}_{642 / 443}^{c}$ (middle), and with the $S A L T 2$ parameters $x_{1}$ and $c$ (bottom). Gray bands highlight $\pm 1 \sigma$ scatter. Intrinsically bright SN 1991T-like and SN 1999aa-like SNe are highlighted in black; highly dust-extincted SNe are highlighted with red squares.

result for $\mathcal{R}_{642 / 443}$ when using SALT2 $m_{B}$ is shown in Fig. 3 (top). The standard deviation of the residual scatter is $\sigma\left(\Delta \mu_{B}\right)=$ $0.128 \pm 0.012 \mathrm{mag}$ when using only $\mathcal{R}_{642 / 443}$ to standardize the peak magnitude $m_{B}$, compared with $0.161 \pm 0.015$ mag when using $\mu_{B}=\left(m_{B}-M\right)+\alpha x_{1}-\beta c$ instead (Fig. 3 bottom). In this case, $\alpha$ and $\beta$ are allowed to vary in the fit.

Synthesizing $m_{B}$ directly from the flux-calibrated near-max spectra provides a peak magnitude estimate independent of a light curve model. In this case $\sigma\left(\Delta \mu_{B}\right)=0.125 \pm 0.011 \mathrm{mag}$ when measuring $m_{B}$ and $\mathcal{R}_{642 / 443}$ from the same spectra. Note that this standardization is achieved using only a single flux calibrated spectrum per $\mathrm{SN}$; the light curve fit is used only to determine the phase. This demonstrates the standardization power of a single spectrum and that these flux ratios are not merely correcting for some anomaly of the SALT2 light curve fit.

In Fig. 3, intrinsically bright SN 1991T-like and SN 1999aalike $\mathrm{SNe}$ are highlighted in black; highly extincted $\mathrm{SNe}$ are highlighted with red squares. $\mathcal{R}_{642 / 443}$ provides an effective correction for both of these extremes for which $x_{1}$ and $c$ are less effective.

We also repeated this analysis fitting a combination of color and flux ratios. In this case, we dereddened the spectra using the SALT2 color model so that the resulting spectra have $E(B-V)=$ 0 ; this removes color from both extrinsic dust reddening as well as possible intrinsic color without trying to distinuish between the two effects. The remaining spectral variations, however, should be predominantly due to small-scale intrinsic effects and not extrinsic dust reddening. We denote flux ratios from these decolored spectra as $\mathcal{R}^{c}$. Their correlation with color-corrected Hubble diagram residuals is shown in the lower-right triangle of Fig. 1; the final selection was based upon the combined fit of $c$ and $\mathcal{R}^{c}$, not just the correlations in Fig. 1. Several combinations of $c$ and $\mathcal{R}^{c}$ have correlations with absolute magnitude of $\sim 0.95$ and result in $\sigma\left(\Delta \mu_{B}\right) \sim 0.12 \mathrm{mag}$. Figure 3 (middle) shows the results for $\mathcal{R}_{642 / 443}^{c}$ with $\sigma\left(\Delta \mu_{B}\right)=0.119 \pm 0.011$ mag. Adding the light curve shape parameter $x_{1}$ to the fits does not improve the results for the single- $\mathcal{R}$ fits or the $c, \mathcal{R}^{c}$ fits.

The results are summarized in Table 1 . Table 2 in the online version lists the supernovae used with their redshifts, $x_{1}, c, \Delta \mu_{B}$, and $\mathcal{R}^{(c)}$ values. For all selected ratios, a single flux ratio results in smaller or competitive Hubble diagram residual scatter compared to what is acheived by standard corrections for light curve shape and color $\left(x_{1}\right.$ and $\left.c\right)$; the combination of a color-corrected ratio $\mathcal{R}^{c}$ and $c$ consistently outperforms $x_{1}$ and $c$. The dispersion of the core is also improved; flux ratios are not merely correcting a few outliers. The largest discrepancy between the training and validation results is for $\mathcal{R}_{641 / 417}$, but even in this case, the validation set has a residual scatter of only $0.185 \pm 0.025 \mathrm{mag}$. The fits are error weighted but the standard deviation is not, which allows some cases where the combined fit has a scatter either less than or greater than the scatter of both of the individual fits.

\section{Discussion}

The 130 to $330 \mathrm{~nm}$ wavelength baseline of the flux ratios means that they are effectively color measurements using very narrow band filters, thus they can correct for extrinsic dust extinction in a manner similar to standard $E(B-V)$ corrections. In fact, $\mathcal{R}_{642 / 443}$ has a correlation of 0.83 with $E(B-V)$ and four of the five $\mathrm{SNe}$ with the largest $\mathcal{R}_{642 / 443}$ values have host galaxy ISM features in their observed spectra, indicating extrinsic dust extinction.

However, $\mathcal{R}_{642 / 443}$ also appears sensitive to intrinsic $\mathrm{SNe}$ variations that are correlated with absolute magnitude. There are other $\operatorname{dim} \mathrm{SNe}$ in this sample with large $\mathcal{R}_{642 / 443}$ and no spectral evidence for host extinction, and $\mathcal{R}_{642 / 443}$ is able to standardize absolute magnitudes better than color alone: using $c$ only, the Hubble residuals have a standard deviation of $0.215 \pm 0.020$ mag. Additionally, the characteristic size of the correlation peaks in Fig. 1 are typical of the widths of SN features, indicating a sensitivity to instrinsic variations of the supernovae.

PHOENIX calculations based upon the W7 explosion model (Bongard et al. 2008) show a strong correlation of $\mathcal{R}_{642 / 443}$ with 
the absolute $B$-band magnitude, driven by large intrinsic variations of Fe II and Fe III line blends around $443 \mathrm{~nm}$. The region redward of $640 \mathrm{~nm}$ has relatively little intrinsic variation in these models and serves as a normalization anchor for the ratio. The $\mathcal{R}_{642 / 443}$ correlation also appears in a new survey of 2D SEDONA (Kasen et al. 2006) models spanning nickel mass 0.4-1.0 $M_{\odot}$ with 30 different viewing angles per model; these generally reproduce the known Phillips brighter-broader correlation. The models exhibit a similar correlation between $\mathcal{R}_{642 / 443}$ and absolute magnitude as we found, but the correlation is even stronger than the theoretically modeled Phillips relation (Kasen, private communication). More modeling work is needed to understand the underlying physics of our results.

It may seem surprising that a single parameter could correct for the completely unrelated effects of SN Ia intrinsic variability and extrinsic dust reddening. But note that the slopes for a dust correction and any intrinsic correction will vary with different wavelength pairs selected for the flux ratio. Some wavelength pairs will align the slopes more than others, thus reinforcing the individual corrections and allowing for two physically distinct phenomena to be corrected with a single parameter. The final best choice will also depend upon the strength of the intrinsic correlations for that ratio and how accurately it can be measured.

We checked our results for $\mathcal{R}_{642 / 443}$ using spectra within 2.5 days of maximum from Matheson et al. (2008) after rejecting 3 exhibiting strong $\mathrm{H}-\alpha$ indicative of host galaxy contamination. Uncertainties on $\mathcal{R}_{642 / 443}$ were assigned based on the internal dispersion for the $12 \mathrm{SNe}$ Ia having multiple suitable spectra; otherwise we used uncertainties derived by Matheson et al. (2008) from their comparison with photometry. Corresponding $B$-band peak magnitudes for $16 \mathrm{SNe}$ Ia from Jha et al. (2006) and Hicken et al. (2009) were put on a consistent scale and standardized using $\mathcal{R}_{642 / 443}$, including photometric and redshift errors due to peculiar velocities. With the exception of SN 1999cl, we find good agreement with our results with a consistent slope $\gamma$ and strong correlation $\rho=0.93$ (see Fig. 2). The scatter is $\sigma\left(\Delta \mu_{B}\right)=0.25 \mathrm{mag}(0.22$ for an error weighted $\sigma)$, but the fit has $\chi_{v}^{2}=0.9$, indicating that the dispersion is consistent with the measurement errors for these data and is not a contradiction of our low dispersion result. The environment of SN 1999cl is known to be unusual, having time-varying $\mathrm{Na}$ I $\mathrm{D}$ (Blondin et al. 2009) and "highly nonstandard dust" (Krisciunas et al. 2006). It is also a large outlier ( $1.5 \mathrm{mag}$ ) when corrected by $x_{1}$ and $c$. This exception is consistent with the inability of any single parameter to simultaneously correct intrinsic $\mathrm{SN}$ variations and more than one type of dust behavior.

Application of $\mathcal{R}_{642 / 443}$ requires flux calibrated spectra near maximum with accurate host galaxy subtraction; spectral slope errors should be kept below a few percent. To keep the noise contribution to the Hubble residuals under $0.1 \mathrm{mag}, S / N \gtrsim 25$ in $2000 \mathrm{~km} \mathrm{~s}^{-1}$ bins is sufficient. For distant SNe Ia, where $S / N$ may be a limiting constraint, the spectra may be rebinned to a lower resolution with only a small degradation of the results. For instance, rebinning to $10000 \mathrm{~km} \mathrm{~s}^{-1}$ (still centered on 642 and $443 \mathrm{~nm}$ ) gives $\rho=0.94$ and $\sigma\left(\Delta \mu_{B}\right)=0.145 \mathrm{mag}$. Away from peak brightness the correlations weaken and the standardization slope $\gamma$ changes, but the $5(1+z)$ day window used here is adequate for scheduling spectroscopy based on a pre-maximum lightcurve in any band. Although currently available high redshift SN Ia spectra are insufficient to apply this method, future spectrophotometric instruments in space or on large groundbased telescopes could achieve these requirements and use this method to build an SN Ia Hubble diagram with the potential for significantly improved statistical power and systematics control.

\section{Conclusions}

We have performed a search for spectral flux ratios that correlate with observed variability in SN Ia absolute magnitudes using separate training and validation datasets totaling 58 homogeneously observed SNe with $0.02<z<0.09$. The results reveal single parameter corrections that achieve results better than those from light curve shape and color parameters, even when only a single spectrum is used to measure and standardize the peak magnitude. The combination of a flux ratio and broad-band color provides even better results, significantly outperforming standard corrections for light curve shape and color. The best single flux ratio $\mathcal{R}_{642 / 443}$ results in a Hubble diagram residual scatter of $0.125 \pm 0.011 \mathrm{mag}$; the best combination of color plus a ratio provides $0.119 \pm 0.011 \mathrm{mag}$.

These results highlight the power of spectrophotometric SN Ia observations. Compared to lightcurve shape and color corrections, our flux ratio standardization represents a factor of 1.8 improvement in the statistical weight per SN, and a $35 \%$ improvement in the upper bound on the potential bias from uncorrected SN Ia variability. These results work well for both extrinsic dust extinction and intrinsic varations such as SN 1991Tlike and SN 1999aa-like SNe, and they effectively correct SNe Ia over a range of light curve shapes and colors, host galaxy properties, and intrinsic subtypes, offering the promise that this method will be similarly powerful for future high- $z$ SN Ia observations.

Acknowledgements. We are grateful to the technical and scientific staff of the University of Hawaii 2.2-m telescope, Palomar Observatory, Lick Observatory, and the High Performance Research and Education Network (HPWREN) for their assistance in obtaining these data. We also thank Julien Guy and David Rubin for assistance with light curve fits. This work was supported in France by CNRS/IN2P3, CNRS/INSU, CNRS/PNC, and used the resources of the IN2P3 computer center. This work was also supported in part by the Director, Office of Science, Office of High Energy and Nuclear Physics, of the US Department of Energy (DOE) under Contract Nos. DE-FG02-92ER40704, DE-AC02-05CH11231, and DE-FG02-06ER06-04; by the Director, Office of Science, Office of Advanced Scientific Computing Research, of the US DOE under Contract No. DE-AC02-05CH11231; by a grant from the Gordon \& Betty Moore Foundation; by National Science Foundation Grant Nos. AST-0407297 (QUEST), AST-0606772 (CfA Supernova Archive), and 0087344 \& 0426879 (HPWREN); by a Henri Chretien International Research Grant administrated by the American Astronomical Society; and the France-Berkeley Fund.

\section{References}

Aldering, G., Adam, G., Antilogus, P., et al. 2002, Proc. SPIE, 4836, 61 Blondin, S., Prieto, J. L., Patat, F., et al. 2009, ApJ, 693, 207

Bongard, S., Baron, E., Smadja, G., Branch, D., \& Hauschildt, P. H. 2006, ApJ, 647,513

Bongard, S., Baron, E., Smadja, G., Branch, D., \& Hauschildt, P. H. 2008, ApJ, 687,456

Bronder, T. J., Hook, I. M., Astier, P., et al. 2008, A\&A, 477, 717 Cardelli, J. A., Clayton, G. C., \& Mathis, J. S. 1989, ApJ, 345, 245 Foley, R. J., Filippenko, A. V., \& Jha, S. W. 2008, ApJ, 686, 117 Guy, J., Astier, P., Baumont, S., et al. 2007, A\&A, 466, 11 Hachinger, S., Mazzali, P. A., \& Benetti, S. 2006, MNRAS, 370, 299 Hicken, M., Challis, P., Jha, S., et al. 2009 [arXiv:0901 . 4787] Jha, S., Kirshner, R. P., Challis, P., et al. 2006, AJ, 131, 527 Jha, S., Riess, A. G., \& Kirshner, R. P. 2007, ApJ, 659, 122 Kasen, D., Thomas, R. C., \& Nugent, P. 2006, ApJ, 651, 366 Kowalski, M., Rubin, D., Aldering, G., et al. 2008, ApJ, 686, 749 Krisciunas, K., Prieto, J. L., Garnavich, P. M., et al. 2006, AJ, 131, 1639 Matheson, T., Kirshner, R. P., Challis, P. et al. 2008, AJ, 135, 1598 Nugent, P., Phillips, M., Baron, E., Branch, D., \& Hauschildt, P. 1995, ApJ, 455, L147

Phillips, M. M. 1993, ApJ, 413, L105

Prieto, J. L., Rest, A., \& Suntzeff, N. B. 2006, ApJ, 647, 501

Riess, A. G., \& Livio, M. 2006, ApJ, 648, 884

Riess, A. G., Nugent, P., Filippenko, A. V., Kirshner, R. P., \& Perlmutter, S. 1998, ApJ, 504, 935

Schlegel, D. J., Finkbeiner, D. P., \& Davis, M. 1998, ApJ, 500, 525 


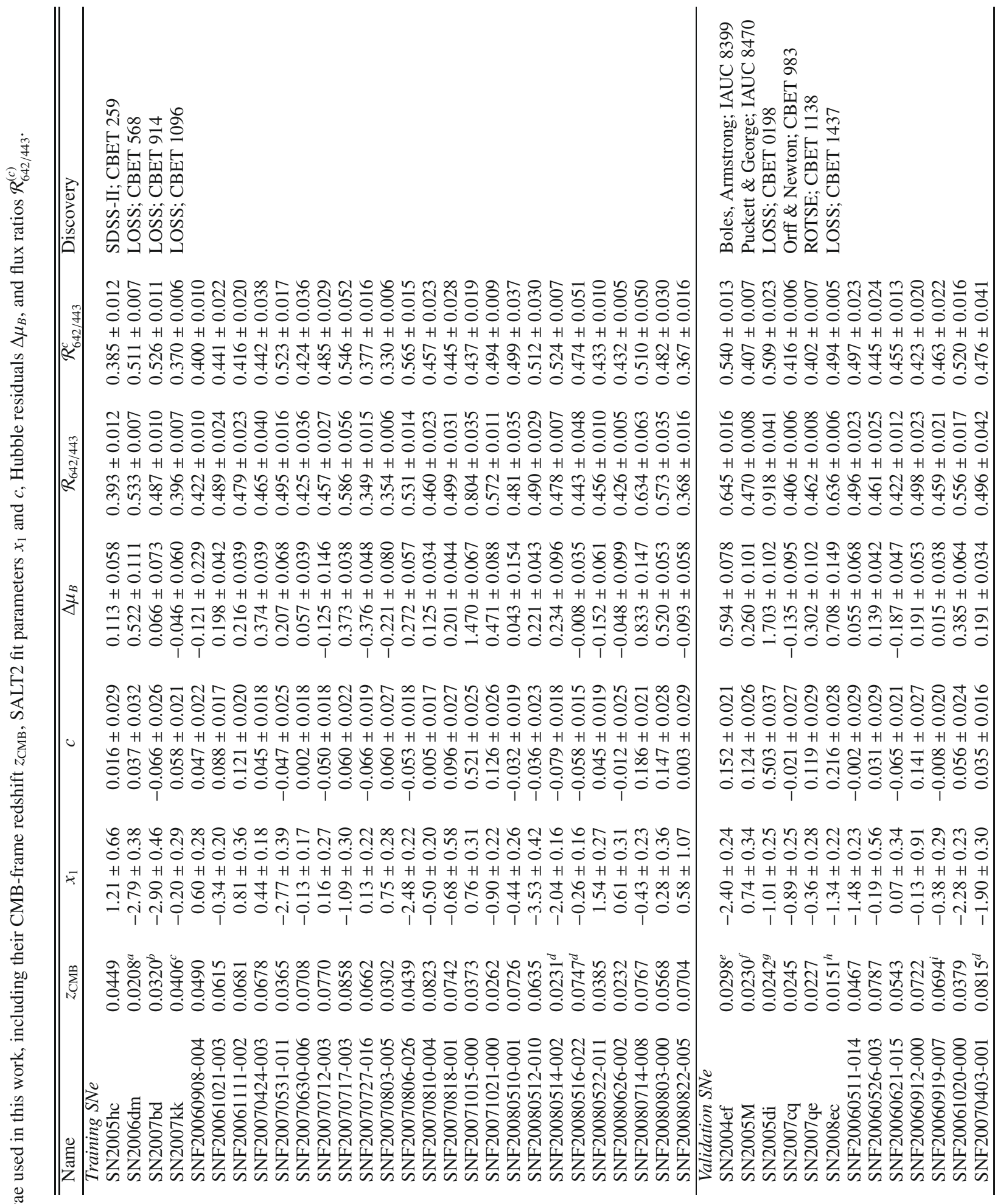


S. Bailey et al. (Nearby Supernova Factory): Using spectral flux ratios to standardize SN Ia luminosities, Online Material p 2

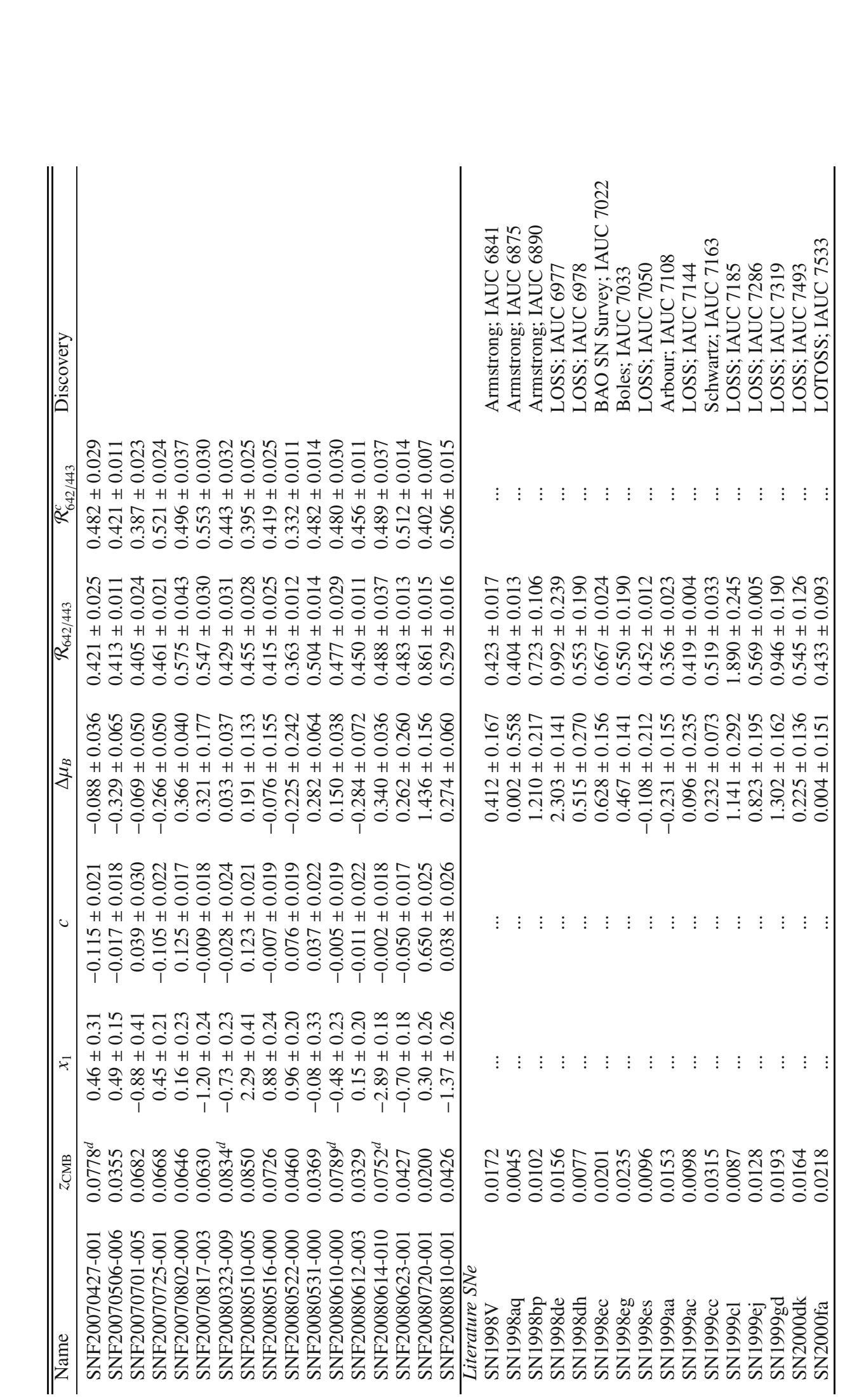

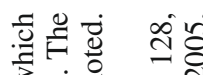

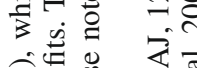

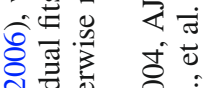

:

$\square . \Xi 5$

政

i

용

"ृष

更

ஸेत्त

d

a

夜定

$\overrightarrow{0}$ ज

吾焉

焉的

永言

专

o

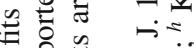

홍

然焉

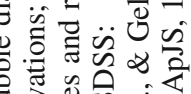

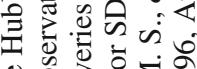
茥 o 记 要究可 的氜 $\overline{2}$ o 责岁.

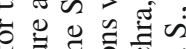

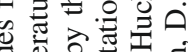

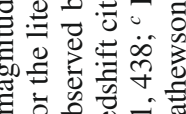

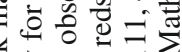

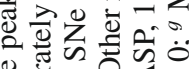

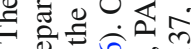
× च $\therefore$ 응

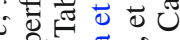

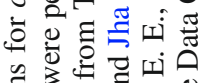
o w 은.

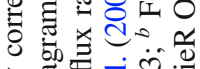

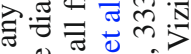
\%ั0

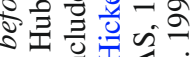

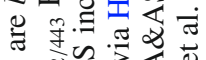
拝已 क्ज़

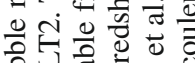

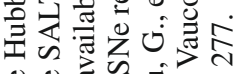

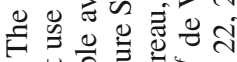

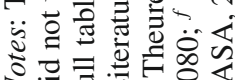

